\title{
Severity of Tomato Late Blight Caused by Phytophthora infestans (Mont.) De Bary in Kashmir
}

\author{
Farheen Chasti, Nazir Ahmad Bhat, Roaf Ahmad Rather*, \\ Masrat Ul Nisar and Tanveer Ahmad Wani
}

Department of Plant Pathology FoA, SKUAST-K, Wadura Sopore, J\&K-193201, India

*Corresponding author

\section{A B S T R A C T}

\begin{tabular}{|l|}
\hline Ke y w or d s \\
$\begin{array}{l}\text { Tomato (Lycopersicon } \\
\text { esculentum Mill), } \\
\text { Phytophthora infestans }\end{array}$ \\
\hline Article Info \\
\hline $\begin{array}{l}\text { Accepted: } \\
\text { 24 September } 2018 \\
\text { Available Online: } \\
\text { 10 October } 2018\end{array}$ \\
\hline
\end{tabular}

\section{Keywords}

Tomato (Lycopersicon esculentum Mill),

\section{Introduction}

Tomato (Lycopersicon esculentum Mill) is one of the most important vegetable crops of the family Solanaceae. It is considered to have originated in Peru Ecuador Bolivia region of the Andes in South America. It is one of the most popular protective foods because of its high lycopene content and is a widely grown vegetable in the world ranking second in importance after potato (Rana, 2008). Because of its high nutritive value it is also most commonly grown vegetable in the kitchen gardens and therefore, called as poor man's orange. It is not only richest source of lycopene, beta-carotene, folate, flavonoids, potassium, vitamin $\mathrm{E}$, vitamin $\mathrm{C}$ and vitamin
Tomato (Lycopersicon esculentum Mill) is one of the most important vegetable crops of the family Solanaceae. Survey for prevalence and severity of late blight of tomato was conducted in three vegetable growing districts of Kashmir valley viz. Anantnag, Baramulla and Budgam. Maximum disease incidence (10.24\%), intensity (3.79\%) and infected fruits (.06\%) were observed in district Anantnag, followed by district Budgam with 9.67\% disease incidence, $3.14 \%$ disease intensity and $3.50 \%$ infected fruits and the least disease incidence $(09.33 \%)$, disease intensity $(3.04 \%)$ and infected fruits $(3.47 \%)$ were recorded in district Baramulla during the first stage of survey. The data revealed that the late blight disease of tomato (Phytophthora infestans) is more or less prevalent in almost every tomato field of Kashmir valley with varied incidence and severity from place to place. 
(Xanthomonas axonopodis pv vesicatoria) and bacterial wilt caused by Ralstonia solanacearum (Naika et al., 2005). Amongst these, late blight caused by Phytopthora infestasns (Mont.) de Bary, is the most destructive disease of tomato and potato in many parts of the world and was responsible for the Irish famine in the middle of the $19^{\text {th }}$ century where over one million died and 1.5 million emigrated (Tsedaley, 2014). It was in France where late blight on tomato was first described by Tulasne in 1854 and is one of the most disturbing diseases of tomatoes and potatoes (Drenth et al., 1995) both in temperate and tropical regions.

\section{Materials and Methods}

\section{Survey}

Survey for prevalence and severity of late blight of tomato was conducted in three vegetable growing districts of Kashmir valley viz. Anantnag, Baramulla and Budgam. Three major tomato growing belts were identified from each of the districts and three locations were randomly selected from each of these belts. Vegetable fields of these locations were visited twice in 2016 at green fruit and ripe fruit stage. Observations were recorded on three randomly selected sites from each location.

\section{Assessment of disease incidence}

A disc was thrown into the tomato fields at each location to randomly select three sites for recording observations. At each site where the disc fell, ten plants were selected and total number and number of leaves infected with late blight was recorded on four branches on four sides representing lower, middle and upper portion of each plant. Disease incidence was calculated using the formula (Meya et al., 2015):

Disease incidence $(\%)=(\mathrm{n} / \mathrm{N}) \times 100$
Where $\mathrm{n}$ is the number of leaves exhibiting late blight symptoms and $\mathrm{N}$ is the total number of leaves observed. Average of ten plants represented the disease incidence of respective sites and average of three sites represented average disease incidence of a locality.

\section{Disease intensity}

Leaves observed for disease were graded according to 0-5 scale given by Sokhi et al., (1993):

$0=$ No disease

$1=\mathrm{Up}$ to $20 \%$ leaf area affected

$2=21-40 \%$ leaf area affected

$3=41-60 \%$ leaf area affected

$4=61-80 \%$ leaf area affected

$5=$ More than $80 \%$ leaf area affected

Sum of the numerical ratings according to the given scale was computed and used to calculate disease intensity by the formula:

Disease intensity $=\frac{\sum \mathrm{nv}}{\mathrm{N} \times \mathrm{G}} \times 100$

Where,

$\sum$ : Summation

v: Disease score

$\mathrm{n}$ : Number of leaves showing a particular score

$\mathrm{N}$ : Number of leaves examined

G: Maximum Score

(Mabbett and Phelps, 1984)

\section{Disease incidence on fruits}

Disease incidence on fruits was recorded and was assessed on the basis of number of fruits available on plants at the time of each survey. Total number of fruits and fruits showing characteristic disease symptoms was counted. Disease incidence on fruits was calculated by using formula: 
$=\frac{\text { Number of diseased fruits }}{\text { Total number of fruits }} \times 100$

\section{Results and Discussion}

\section{Survey for recording disease status}

With a view to find out the status of late blight of tomato in Kashmir valley, survey of important tomato growing belts of district Anantnag, Baramulla and Budgam was conducted during 2016. Incidence and intensity of late blight of tomato was recorded in two stages of survey and the data is presented in Tables 1 and 2 respectively. Observation on fruit infection was also recorded and the cumulative data is presented in Table 3.

\section{Disease incidence}

The data (Table 1) reveals that late blight of tomato was prevalent in all locations surveyed with varying degrees of incidence, which was minimum in first but maximum in second stage of survey. Disease incidence in the first stage of survey, conducted at green fruit stage, ranged between 7.94-13.71 per cent and in second stage of survey, conducted at ripe fruit stage, between 17.47-23.01 per cent, respectively.

Highest disease incidence of 13.71 per cent at first stage of survey was recorded at Sagam, Anantnag, followed by Wagoora, Budgam (12.48\%), Lalan, Anantnag (11.72\%), Larnoo, Anantnag (11.33\%), Haji Danter, Anantnag (10.87\%), Nehalpora, Baramulla (10.29\%) and Larkipora, Anantnag (9.99\%), respectively. During second stage of survey, the disease incidence at these locations was 19.61, 20.68, 22.30, 19.38, 22.05, 20.78 and 23.01 per cent, respectively. While highest disease incidence of 23.01per cent was recorded, during second stage of survey, at Larkipora, Anantnag followed by Mehmoodaabad, Anantnag (22.58\%), Chakla, Baramulla (22.57\%), Kaisarmulla, Budgam (22.33\%), Lalan, Anantnag (22.30\%), Haji Danter, Anantnag (22.05\%) and HilarArhama, Anantnag (21.67\%), respectively. Other localities, which followed in decreasing order of disease incidence. were Nowbugh, Wusan, UttarasooNajigund, Wager, Arampora, Stadium Colony and Kremshore, respectively, while in second stage, Shangrin, UttarasooNajigund, Nehalpora, Wusan, Chinkipora, Stadium colony and Nowbugh, respectively. Least disease incidence of 07.94 per cent was recorded at Shangrin (Anantnag) at green fruit stage and 17.47 per cent was recorded at Bugam (Budgam) at ripe fruit stage.

Amongst the districts, the highest average disease incidence at green fruit stage was recorded in district Anantnag (10.24\%) followed by Budgam (09.67\%) and Baramulla $(09.33 \%)$, respectively where as in second stage of survey, highest disease incidence was again recorded in district Anantnag (21.43\%), followed by Budgam $(20.01 \%)$ and Baramulla $(19.4 \%)$, respectively. The average of disease incidence of all the districts was $09.75 \%$ at the green fruit stage and $20.46 \%$ at ripe fruit stage, respectively.

\section{Disease intensity}

The data presented in Table 2 reveals that intensity of late blight of tomato varied at all locations surveyed and was minimum in first but maximum in second stage of survey. Disease intensity in the first stage of survey, conducted at green fruit stage ranged between 2.13-5.10 per cent and in second stage of survey, conducted at ripe fruit stage between 7.13-10.19 per cent, respectively.

Highest disease intensity of 5.10 per cent at 
first stage of survey was recorded at UttarasoNajigund, Anantnag, followed by Lalan, Anantnag (4.78\%), Sagam, Anantnag (4.61\%), Haji Danter, Anantnag (4.78\%), Wagoora, Budgam (4.41\%), Kremshore, Budgam (3.74\%) and Wager, Budgam (3.59\%), respectively. During second stage of survey, the disease intensity at these location was $9.02,9.98,8.13,9.58,8.72,8.85$ and 8.57 per cent, respectively.

While highest disease intensity of 10.19 per cent was recorded during second stage of survey at HilarArahama, Anantnag, Dawlatpora, Budgam (10.17\%), Larkipora, Anantnag (10.15\%), Mehmoodaabad, Anantnag (10.14\%), Lalan, Anantnag (09.98\%), Nowbugh, Budgam $(09.72 \%)$ and Wusan, Baramulla (09.68\%), respectively.

Other localities, which followed in decreasing order of disease intensity Larnoo, Wusan, Larkipora, Nehalpora, Nowbugh, Palhalan and Stadium colony, respectively, while in second stage, Haji danter, Khansahib, Kuthipora, Shangrin, Stadium colony, Kaisarmulla and Chakla, respectively. Least disease intensity of 2.13 per cent was recorded at Kaisarmulla (Budgam) at green fruit stage and 7.13 per cent was recorded at Palhalan, Baramulla at ripe fruit stage.

Amongst the districts, the highest average disease intensity was recorded in district Anantnag $(3.79 \%)$ followed by district Budgam (3.14\%) and district Baramulla $(3.04 \%)$ at green fruit stage where as in second stage of survey, highest disease intensity was again recorded in district Anantnag (9.37\%), followed by district Budgam (9.05\%) and district Baramulla $(8.48 \%)$, respectively. The average disease intensity of all the districts was $3.32 \%$ at the green fruit stage and $8.97 \%$ at ripe fruit stage, respectively.
Fruit infection, unmarketable culls and fruit yield

Data presented in Tables 3 and 4 reveals that some of the fruits present on plants at first and second stage of survey, the surveyed fields exhibited symptoms of late blight disease out of which, a good number of fruits were infected to the extent that these could be graded as unmarketable culls. Infected fruits ranged from 2.14-5.08 and 5.95-9.35 per cent during first and second stage of survey, respectively. At second stage of survey many infected fruits had rotten to the extent that these were categorized as unmarketable culls and ranged between 21.16-27.39\%.

During first stage of survey highest infected fruits $(5.08 \%)$ were recorded at Hilar Arhama, Anantnag, while least infected fruits during first stage of survey were recorded at Palhalan, Baramulla (2.14\%). During second stage of survey maximum infected fruits $(9.35 \%)$ were recorded at Haji Danter, Anantnag, while in minimum at Arampora, Baramulla (5.95\%). Maximum infected fruits categorized as unmarketable culls during second stage of survey were recorded at Wagoora, Budgam $(27.39 \%)$ and least unmarketable culls were at Wadura, Baramulla (21.16\%).

Perusal of the data (Table 5) reveals that fruit yield ranged from 140-190 $\mathrm{q} \mathrm{ha}^{-1}$ in the surveyed fields. Highest fruit yield of $190 \mathrm{q}$ $\mathrm{ha}^{-1}$ was recorded at Wadura, while as lowest yield was recorded at Wagoora, Budgam (140 $\mathrm{q} \mathrm{ha}{ }^{-1}$ ).

Data on overall scenario of disease parameters amongst the surveyed districts at green fruit stage reveals that maximum disease incidence $(10.24 \%)$, intensity $(3.79 \%)$ and infected fruits (4.06\%) were observed in district Anantnag, followed by district Budgam with $9.67 \%$ disease incidence, $3.14 \%$ disease intensity and $3.50 \%$ infected fruits. 
Table.1 Incidence (\%) of late blight of tomato (Phytophthora infestans) on leaves at various locations of Kashmir

\begin{tabular}{|c|c|c|c|c|c|c|c|c|}
\hline \multirow[t]{2}{*}{ Districts } & \multirow[t]{2}{*}{ Belts } & \multirow[t]{2}{*}{ Locations } & \multirow{2}{*}{$\begin{array}{l}\text { Green } \\
\text { fruit } \\
\text { stage }\end{array}$} & \multicolumn{2}{|c|}{ C.I $(95 \%)$} & \multirow{2}{*}{$\begin{array}{l}\text { Ripe } \\
\text { fruit } \\
\text { stage }\end{array}$} & \multicolumn{2}{|c|}{ C.I $(95 \%)$} \\
\hline & & & & $\begin{array}{l}\text { Lower } \\
\text { limit }\end{array}$ & $\begin{array}{l}\text { Upper } \\
\text { limit }\end{array}$ & & $\begin{array}{l}\text { Lower } \\
\text { limit }\end{array}$ & $\begin{array}{l}\text { Upper } \\
\text { limit }\end{array}$ \\
\hline \multirow[t]{13}{*}{ Anantnag } & \multirow[t]{4}{*}{ Achabal } & Haji Danter & 10.87 & & & 22.05 & & \\
\hline & & UttarasooNajigund & 09.74 & & & 20.91 & & \\
\hline & & Lalan & 11.72 & & & 22.30 & & \\
\hline & & Sub Mean & 10.78 & 09.66 & 11.90 & 21.75 & 20.59 & 22.59 \\
\hline & \multirow[t]{4}{*}{ Breng } & Sagam & 13.71 & & & 19.61 & & \\
\hline & & Larnoo & 11.33 & & & 19.38 & & \\
\hline & & HilarArhama & 8.92 & & & 21.67 & & \\
\hline & & Sub Mean & 11.32 & 08.50 & 14.02 & 20.22 & 18.79 & 21.65 \\
\hline & \multirow[t]{4}{*}{ Dooru } & Larkipora & 9.99 & & & 23.01 & & \\
\hline & & Shangrin & 7.94 & & & 21.34 & & \\
\hline & & Mehmooabad & 7.95 & & & 22.58 & & \\
\hline & & Sub Mean & 8.63 & 07.45 & 09.97 & 22.31 & 21.32 & 23.30 \\
\hline & & Mean & 10.24 & 08.63 & 11.85 & 21.43 & 20.21 & 22.65 \\
\hline \multirow[t]{13}{*}{ Baramulla } & \multirow[t]{4}{*}{ Sopore } & Arampora & 09.60 & & & 19.44 & & \\
\hline & & Chinkipora & 09.07 & & & 20.45 & & \\
\hline & & Wadura & 09.18 & & & 18.8 & & \\
\hline & & Sub Mean & 09.28 & 08.96 & 09.60 & 19.56 & 18.62 & 20.50 \\
\hline & \multirow[t]{4}{*}{ Pattan } & Palhalan & 09.02 & & & 17.66 & & \\
\hline & & Wusan & 09.85 & & & 20.47 & & \\
\hline & & Nehalpora & 10.29 & & & 20.78 & & \\
\hline & & Sub Mean & 09.72 & 08.99 & 10.45 & 19.64 & 17.70 & 21.58 \\
\hline & \multirow{4}{*}{$\begin{array}{c}\text { Baramulla } \\
\text { (Tehsil) }\end{array}$} & Stadium Colony & 09.56 & & & 20.25 & & \\
\hline & & Johama & 08.20 & & & 19.96 & & \\
\hline & & Chakla & 09.21 & & & 22.57 & & \\
\hline & & Sub Mean & 08.99 & 08.19 & 09.79 & 20.63 & 19.01 & 22.25 \\
\hline & & Mean & 09.33 & 08.91 & 10.25 & 19.94 & 19.27 & 20.61 \\
\hline \multirow[t]{14}{*}{ Budgam } & \multirow[t]{4}{*}{ Khansahib } & Kremshore & 09.50 & & & 19.00 & & \\
\hline & & Khansahib & 08.82 & & & 19.94 & & \\
\hline & & Wager & 09.73 & & & 19.21 & & \\
\hline & & Sub Mean & 09.35 & 08.82 & 09.88 & 19.38 & 18.85 & 19.91 \\
\hline & \multirow[t]{4}{*}{ Chadoora } & Bugam & 09.75 & & & 17.47 & & \\
\hline & & Kaisarmulla & 08.29 & & & 22.33 & & \\
\hline & & Nowbugh & 09.88 & & & 20.22 & & \\
\hline & & Sub Mean & 09.31 & 08.36 & 10.26 & 20.01 & 17.25 & 22.77 \\
\hline & \multirow[t]{4}{*}{ B.K Pora } & Kuthipora & 08.94 & & & 20.73 & & \\
\hline & & Wagoora & 12.48 & & & 20.68 & & \\
\hline & & Dawlatpore & 09.63 & & & 20.48 & & \\
\hline & & Sub Mean & 10.35 & 08.22 & 12.48 & 20.63 & 20.49 & 20.77 \\
\hline & \multirow{2}{*}{\multicolumn{2}{|c|}{$\begin{array}{l}\text { Mean } \\
\text { Overall Mean }\end{array}$}} & 09.67 & 09.00 & 10.34 & 20.01 & 19.30 & 20.72 \\
\hline & & & 09.75 & 09.23 & 10.27 & 20.46 & 19.62 & 21.30 \\
\hline
\end{tabular}


Table.2 Intensity (\%) of late blight of tomato (Phytopthora infestans) on leaves at various location of Kashmir

\begin{tabular}{|c|c|c|c|c|c|c|c|c|}
\hline \multirow[t]{2}{*}{ Districts } & \multirow[t]{2}{*}{ Belts } & \multirow[t]{2}{*}{ Locations } & \multirow{2}{*}{$\begin{array}{l}\text { Green } \\
\text { fruit } \\
\text { stage }\end{array}$} & \multicolumn{2}{|c|}{ C.I $(95 \%)$} & \multirow{2}{*}{$\begin{array}{l}\text { Ripe } \\
\text { fruit } \\
\text { stage }\end{array}$} & \multicolumn{2}{|c|}{ C.I $(95 \%)$} \\
\hline & & & & $\begin{array}{c}\text { Lower } \\
\text { limit }\end{array}$ & $\begin{array}{c}\text { Upper } \\
\text { limit }\end{array}$ & & $\begin{array}{c}\text { Lower } \\
\text { limit }\end{array}$ & $\begin{array}{c}\text { Upper } \\
\text { limit }\end{array}$ \\
\hline \multirow[t]{13}{*}{ Anantnag } & \multirow[t]{4}{*}{ Achabal } & Haji Danter & 04.58 & & & 09.58 & & \\
\hline & & UttarasooNajigund & 05.10 & & & 09.02 & & \\
\hline & & Lalan & 04.78 & & & 09.98 & & \\
\hline & & Sub Mean & 04.82 & 04.53 & 05.11 & 09.53 & 08.99 & 10.07 \\
\hline & \multirow[t]{4}{*}{ Breng } & Sagam & 04.61 & & & 08.13 & & \\
\hline & & Larnoo & 03.68 & & & 07.98 & & \\
\hline & & HilarArhama & 02.69 & & & 10.19 & & \\
\hline & & Sub Mean & 03.66 & 02.75 & 04.75 & 08.77 & 07.38 & 10.16 \\
\hline & \multirow{4}{*}{ Dooru } & Larkipora & 03.47 & & & 10.15 & & \\
\hline & & Shangrin & 02.43 & & & 09.12 & & \\
\hline & & Mehmooabad & 02.74 & & & 10.14 & & \\
\hline & & Sub Mean & 02.88 & 02.28 & 03.48 & 09.80 & 07.92 & 09.26 \\
\hline & & Mean & 03.79 & 02.68 & 04.90 & 09.37 & 08.77 & 09.97 \\
\hline \multirow[t]{13}{*}{ Baramulla } & \multirow[t]{4}{*}{ Sopore } & Arampora & 03.06 & & & 07.91 & & \\
\hline & & Chinkipora & 02.86 & & & 07.95 & & \\
\hline & & Wadura & 02.97 & & & 08.04 & & \\
\hline & & Sub Mean & 02.96 & 02.85 & 03.07 & 07.97 & 07.90 & 08.04 \\
\hline & \multirow[t]{4}{*}{ Pattan } & Palhalan & 03.13 & & & 07.13 & & \\
\hline & & Wusan & 03.50 & & & 09.68 & & \\
\hline & & Nehalpora & 03.26 & & & 08.95 & & \\
\hline & & Sub Mean & 03.29 & 03.07 & 03.51 & 08.59 & 07.11 & 10.07 \\
\hline & \multirow{4}{*}{$\begin{array}{c}\text { Baramulla } \\
\text { (Tehsil) }\end{array}$} & Stadium Colony & 03.13 & & & 09.08 & & \\
\hline & & Johama & 02.62 & & & 08.49 & & \\
\hline & & Chakla & 02.90 & & & 09.03 & & \\
\hline & & Sub Mean & 02.88 & 02.59 & 03.17 & 08.87 & 08.50 & 09.24 \\
\hline & & Mean & 03.04 & 02.79 & 03.29 & 08.48 & 07.96 & 09.00 \\
\hline \multirow[t]{13}{*}{ Budgam } & \multirow[t]{4}{*}{ Khansahib } & Kremshore & 03.74 & & & 08.85 & & \\
\hline & & Khansahib & 02.37 & & & 09.26 & & \\
\hline & & Wager & 03.59 & & & 08.57 & & \\
\hline & & Sub Mean & 03.23 & 02.83 & 04.08 & 08.89 & 09.28 & 08.50 \\
\hline & \multirow[t]{4}{*}{ Chadoora } & Bugam & 03.04 & & & 07.87 & & \\
\hline & & Kaisarmulla & 02.13 & & & 09.06 & & \\
\hline & & Nowbugh & 03.26 & & & 09.72 & & \\
\hline & & Sub Mean & 02.81 & 02.14 & 03.48 & 08.88 & 07.82 & 09.94 \\
\hline & \multirow[t]{4}{*}{ B.K Pora } & Kuthipora & 02.73 & & & 09.22 & & \\
\hline & & Wagoora & 04.41 & & & 08.72 & & \\
\hline & & Dawlatpore & 03.00 & & & 10.17 & & \\
\hline & & Sub Mean & 03.38 & 02.36 & 04.40 & 09.37 & 08.53 & 10.21 \\
\hline & \multirow{2}{*}{\multicolumn{2}{|c|}{$\begin{array}{l}\text { Mean } \\
\text { Overall Mean }\end{array}$}} & 03.14 & 02.81 & 03.47 & 09.05 & 08.73 & 09.37 \\
\hline & & & 3.32 & 02.89 & 03.75 & 8.97 & 08.46 & 09.48 \\
\hline
\end{tabular}


Table.3 Incidence (\%) of tomato fruit infection by Phytophthora infestans at Various locations of Kashmir

\begin{tabular}{|c|c|c|c|c|c|c|c|c|}
\hline \multirow[t]{2}{*}{ Districts } & \multirow[t]{2}{*}{ Belts } & \multirow[t]{2}{*}{ Locations } & \multirow{2}{*}{$\begin{array}{l}\text { Green } \\
\text { fruit } \\
\text { stage }\end{array}$} & \multicolumn{2}{|c|}{ C.I $(95 \%)$} & \multirow{2}{*}{$\begin{array}{l}\text { Ripe } \\
\text { fruit } \\
\text { stage }\end{array}$} & \multicolumn{2}{|c|}{ C.I $(95 \%)$} \\
\hline & & & & $\begin{array}{l}\text { Lower } \\
\text { limit }\end{array}$ & $\begin{array}{l}\text { Upper } \\
\text { limit }\end{array}$ & & $\begin{array}{l}\text { Lower } \\
\text { limit }\end{array}$ & $\begin{array}{l}\text { Upper } \\
\text { limit }\end{array}$ \\
\hline \multirow[t]{13}{*}{ Anantnag } & \multirow[t]{4}{*}{ Achabal } & Haji Danter & 02.17 & & & 09.35 & & \\
\hline & & $\begin{array}{c}\text { UttarasooNajigun } \\
\mathrm{d}\end{array}$ & 04.37 & & & 08.26 & & \\
\hline & & Lalan & 04.04 & & & 07.73 & & \\
\hline & & Sub Mean & 03.53 & 02.54 & 08.45 & 08.45 & 07.51 & 09.39 \\
\hline & \multirow[t]{4}{*}{ Breng } & Sagam & 04.35 & & & 07.51 & & \\
\hline & & Larnoo & 03.81 & & & 08.21 & & \\
\hline & & HilarArhama & 05.08 & & & 07.91 & & \\
\hline & & Sub Mean & 04.41 & 03.68 & 07.88 & 07.88 & 08.27 & 07.49 \\
\hline & \multirow[t]{4}{*}{ Dooru } & Larkipora & 04.80 & & & 06.66 & & \\
\hline & & Shangrin & 04.37 & & & 07.78 & & \\
\hline & & Mehmooabad & 03.51 & & & 07.08 & & \\
\hline & & Sub Mean & 04.23 & 03.48 & 07.17 & 07.17 & 06.27 & 07.82 \\
\hline & & Mean & 04.06 & 03.54 & 04.58 & $\mathbf{0 7 . 8 3}$ & 07.10 & 08.56 \\
\hline \multirow[t]{13}{*}{ Baramulla } & \multirow{4}{*}{ Sopore } & Arampora & 03.37 & & & 05.95 & & \\
\hline & & Chinkipora & 03.11 & & & 07.31 & & \\
\hline & & Wadura & 04.50 & & & 06.56 & & \\
\hline & & Sub Mean & 03.66 & 02.82 & 06.61 & 06.61 & 05.84 & 07.38 \\
\hline & \multirow[t]{4}{*}{ Pattan } & Palhalan & 02.14 & & & 06.38 & & \\
\hline & & Wusan & 04.18 & & & 07.58 & & \\
\hline & & Nehalpora & 04.59 & & & 08.23 & & \\
\hline & & Sub Mean & 03.64 & 02.16 & 07.39 & 07.39 & 06.33 & 08.45 \\
\hline & \multirow{4}{*}{$\begin{array}{c}\text { Baramulla } \\
\text { (Tehsil) }\end{array}$} & Stadium Colony & 02.91 & & & 07.55 & & \\
\hline & & Johama & 02.38 & & & 06.74 & & \\
\hline & & Chakla & 04.02 & & & 07.38 & & \\
\hline & & Sub Mean & 03.10 & 02.15 & 07.22 & 07.22 & 06.73 & 07.69 \\
\hline & & Mean & 03.47 & 03.11 & 03.83 & 07.07 & 06.61 & 07.53 \\
\hline \multirow[t]{14}{*}{ Budgam } & \multirow[t]{4}{*}{ Khansahib } & Kremshore & 02.69 & & & 06.99 & & \\
\hline & & Khansahib & 03.51 & & & 06.94 & & \\
\hline & & Wager & 03.01 & & & 06.74 & & \\
\hline & & Sub Mean & 03.07 & 02.21 & 06.89 & 06.89 & 06.74 & 07.04 \\
\hline & \multirow[t]{4}{*}{ Chadoora } & Bugam & 04.26 & & & 06.63 & & \\
\hline & & Kaisarmulla & 03.99 & & & 07.06 & & \\
\hline & & Nowbugh & 04.23 & & & 07.03 & & \\
\hline & & Sub Mean & 04.16 & 03.99 & 06.91 & 06.91 & 06.64 & 07.18 \\
\hline & \multirow[t]{4}{*}{ B.K Pora } & Kuthipora & 03.55 & & & 08.60 & & \\
\hline & & Wagoora & 03.04 & & & 07.18 & & \\
\hline & & Dawlatpore & 03.24 & & & 07.18 & & \\
\hline & & Sub Mean & 03.28 & 02.99 & 07.65 & 07.65 & 06.27 & 8.58 \\
\hline & \multirow{2}{*}{\multicolumn{2}{|c|}{$\begin{array}{l}\text { Mean } \\
\text { Overall Mean }\end{array}$}} & 03.50 & 02.84 & 04.16 & 07.15 & 06.66 & 07.64 \\
\hline & & & 03.68 & 03.31 & 04.05 & 0.7 .35 & 06.87 & 07.83 \\
\hline
\end{tabular}


Table.4 Unmarketable culls (\%) at various locations of Kashmir during 2016

\begin{tabular}{|c|c|c|c|c|c|}
\hline \multirow[t]{2}{*}{ Districts } & \multirow[t]{2}{*}{ Belts } & \multirow[t]{2}{*}{ Locations } & \multirow{2}{*}{$\begin{array}{l}\text { Unmarketable } \\
\text { culls at Ripe } \\
\text { fruit stage }\end{array}$} & \multicolumn{2}{|c|}{ C.I (95\%) } \\
\hline & & & & Lower Limit & Upper Limit \\
\hline \multirow[t]{13}{*}{ Anantnag } & \multirow[t]{4}{*}{ Achabal } & Haji Danter & $24.51^{*}$ & & \\
\hline & & UttarasooNajigund & 21.25 & & \\
\hline & & Lalan & 24.37 & & \\
\hline & & Sub mean & 23.38 & 21.30 & 25.46 \\
\hline & \multirow[t]{4}{*}{ Breng } & Sagam & 24.37 & & \\
\hline & & Larnoo & 25.88 & & \\
\hline & & HilarArhama & 24.87 & & \\
\hline & & Sub mean & 25.04 & 24.17 & 25.91 \\
\hline & \multirow[t]{4}{*}{ Dooru } & Larkipora & 23.75 & & \\
\hline & & Shangrin & 25.84 & & \\
\hline & & Mehmooabad & 22.92 & & \\
\hline & & Sub mean & 24.17 & 22.50 & 25.84 \\
\hline & & Mean & 24.19 & 23.25 & 25.13 \\
\hline \multirow[t]{13}{*}{ Baramulla } & \multirow[t]{4}{*}{ Sopore } & Arampora & 21.79 & & \\
\hline & & Chinkipora & 24.07 & & \\
\hline & & Wadura & 21.16 & & \\
\hline & & Sub mean & 22.34 & 20.61 & 24.07 \\
\hline & \multirow[t]{4}{*}{ Pattan } & Palhalan & 26.92 & & \\
\hline & & Wusan & 23.19 & & \\
\hline & & Nehalpora & 26.03 & & \\
\hline & & Sub mean & 25.38 & 23.19 & 27.57 \\
\hline & \multirow{4}{*}{$\begin{array}{c}\text { Baramulla } \\
\text { (Tehsil) }\end{array}$} & Stadium colony & 24.74 & & \\
\hline & & Johama & 25.71 & & \\
\hline & & Chakla & 22.28 & & \\
\hline & & Sub mean & 24.24 & 22.24 & 26.24 \\
\hline & & Mean & 23.99 & 22.25 & 25.73 \\
\hline \multirow[t]{13}{*}{ Budgam } & \multirow[t]{4}{*}{ Khansahib } & Kremshore & 23.19 & & \\
\hline & & Khansahib & 26.61 & & \\
\hline & & Wager & 22.14 & & \\
\hline & & Sub mean & 23.98 & 21.33 & 26.63 \\
\hline & \multirow[t]{4}{*}{ Chadoora } & Bugam & 22.28 & & \\
\hline & & Kaisarmulla & 23.19 & & \\
\hline & & Nowbugh & 23.46 & & \\
\hline & & Sub mean & 22.98 & 22.28 & 23.68 \\
\hline & \multirow[t]{4}{*}{ B.K Pora } & Kuthipora & 23.01 & & \\
\hline & & Wagoora & 27.39 & & \\
\hline & & Dawlatpore & 25.27 & & \\
\hline & & Sub mean & 25.22 & 22.74 & 27.70 \\
\hline & \multirow{2}{*}{\multicolumn{2}{|c|}{ A verage }} & 24.06 & 22.79 & 25.35 \\
\hline & & & 24.08 & 23.97 & 24.19 \\
\hline
\end{tabular}

"Out of infected fruits 
Table.5 Tomato fruit yield at various locations of Kashmir during 2016

\begin{tabular}{|c|c|c|c|c|c|}
\hline \multirow[t]{2}{*}{ Districts } & \multirow[t]{2}{*}{ Belts } & \multirow[t]{2}{*}{ Locations } & \multirow{2}{*}{$\begin{array}{l}\text { Unmarketable } \\
\text { culls at Ripe } \\
\text { fruit stage }\end{array}$} & \multicolumn{2}{|c|}{ C.I $(95 \%)$} \\
\hline & & & & Lower Limit & Upper Limit \\
\hline \multirow[t]{13}{*}{ Anantnag } & \multirow[t]{4}{*}{ Achabal } & Haji Danter & 168 & & \\
\hline & & UttarasooNajigund & 177 & & \\
\hline & & Lalan & 162 & & \\
\hline & & Sub mean & 169 & 160.45 & 177.55 \\
\hline & \multirow[t]{4}{*}{ Breng } & Sagam & 155 & & \\
\hline & & Larnoo & 157 & & \\
\hline & & HilarArhama & 151 & & \\
\hline & & Sub mean & 154.33 & 150.86 & 157.80 \\
\hline & \multirow[t]{4}{*}{ Dooru } & Larkipora & 148 & & \\
\hline & & Shangrin & 143 & & \\
\hline & & Mehmooabad & 158 & & \\
\hline & & Sub mean & 149.67 & 141.01 & 158.33 \\
\hline & & Mean & 157.67 & 146.24 & 169.10 \\
\hline \multirow[t]{14}{*}{ Baramulla } & \multirow[t]{4}{*}{ Sopore } & Arampora & 188 & & \\
\hline & & Chinkipora & 180 & & \\
\hline & & Wadura & 190 & & \\
\hline & & Sub mean & 186 & 180.01 & 191.99 \\
\hline & \multirow[t]{4}{*}{ Pattan } & Palhalan & 168 & & \\
\hline & & Wusan & 176 & & \\
\hline & & Nehalpora & 162 & & \\
\hline & & Sub mean & 168.67 & 160.72 & 176.62 \\
\hline & \multirow{4}{*}{$\begin{array}{l}\text { Baramulla } \\
\text { (Tehsil) }\end{array}$} & Stadium colony & 163 & & \\
\hline & & Johama & 158 & & \\
\hline & & Chakla & 182 & & \\
\hline & & Sub mean & 167.70 & 153.36 & 182.04 \\
\hline & \multirow{5}{*}{ Khansahib } & Mean & 174.12 & 162.43 & 185.81 \\
\hline & & Kremshore & 182 & & \\
\hline \multirow[t]{13}{*}{ Budgam } & & Khansahib & 164 & & \\
\hline & & Wager & 188 & & \\
\hline & & Sub mean & 178 & 163.87 & 192.13 \\
\hline & \multirow[t]{4}{*}{ Chadoora } & Bugam & 174 & & \\
\hline & & Kaisarmulla & 166 & & \\
\hline & & Nowbugh & 153 & & \\
\hline & & Sub mean & 164.33 & 152.34 & 176.32 \\
\hline & \multirow[t]{4}{*}{ B.K Pora } & Kuthipora & 175 & & \\
\hline & & Wagoora & 140 & & \\
\hline & & Dawlatpore & 148 & & \\
\hline & & Sub mean & 154.33 & 133.56 & 175.10 \\
\hline & \multirow{2}{*}{\multicolumn{2}{|c|}{$\begin{array}{l}\text { Mean } \\
\text { Overall Mean }\end{array}$}} & 165.55 & 152.09 & 179.01 \\
\hline & & & 165.78 & 156.46 & 175.10 \\
\hline
\end{tabular}


Least disease incidence $(09.33 \%)$, disease intensity (3.04\%) and infected fruits (3.47\%) were recorded in district Baramulla during the first stage of survey. While during second stage of survey similar trend was observed with $21.43 \%$ disease incidence, $9.37 \%$ disease intensity, $7.83 \%$ infected fruits and $24.19 \%$ unmarketable culls recorded in district Anantnag followed by district Budgam with disease incidence of $20.01 \%$, disease intensity $9.05 \%$, infected fruits $7.15 \%$ and unmarketable culls $24.06 \%$. Least disease incidence of $19.94 \%$, disease intensity $8.48 \%$, infected fruits $7.07 \%$ and unmarketable culls $23.99 \%$ was recorded in district Baramulla at the second stage of survey.

It is evident from survey conducted in 2016 that late blight was, more or less, prevalent in almost every tomato field of Kashmir valley with varied incidence and severity from place to place. The disease caused severe damage to the high yielding exotic variety like Roma and Marglobe. The local variety, which has vernacular name as Kashmir round also showed high level of susceptibility to the disease. Perusal of data at green fruit stage reveals that out of 27 locations surveyed, highest disease incidence of $13.71 \%$ was recorded at Sagam, highest disease intensity $(5.10 \%)$ UttarasoNajigund and highest fruit infection (5.08\%) HilarArhama. Palhalan, Dawlatpora and Wager recorded the disease parameters (disease incidence, intensity and fruit infection) to moderate levels. Least disease incidence $(7.94 \%)$, intensity $(2.13 \%)$ and fruit infection $(2.14 \%)$ were recorded at Shangrin, Kaisarmulla and Palhalan, respectively. Similarly the data revealed that at ripe fruit stage highest disease incidence $(23.01 \%)$, intensity $(10.19 \%)$ and fruit infection $(9.35 \%)$ were recorded at Larkipora, HilarArhama and Haji Danter, respectively. Highest unmarketable culls (27.39\%) and least fruit yield $\left(140 \mathrm{qha}^{-1}\right)$ were recorded at wagoora (Budgam). Kremshore, Wadura,
Nowbugh and Wager recorded the disease parameters (disease incidence, intensity and fruit infection) to moderate levels. Least disease incidence $(17.47 \%)$, intensity $(07.13 \%)$ and fruit infection $(05.95 \%)$ were recorded at Bugam, Palhalan and Arampora, respectively. Least unmarketable cull $(21.16 \%)$ and highest fruit yield $\left(190 \mathrm{~kg}^{-1}\right)$ were recorded at Wadura (Baramulla). Tomato cultivation in Larkipora, HilarArhama, Haji Danter and Wagoora are carried out under humid conditions on flat beds without following the recommended practices of planting, with respect to spacing and staking. Besides this, farmers in Sagam, Uttarasonajigund and HilarArhama follow monoculture of solanacous crops over large contiguous areas. High levels of disease in these areas may probably be due to monoculture of solanacous crops and humid conditions during cropping season. The disease has been reported to be favored by moderate temperature, $12-20^{\circ} \mathrm{C}$ and leaf wetness more than 10 hours and can occur in warm climates, where the nights are cold (Vale et al., 2000). Its prevalence to varying extents in conditions of higher humidity, low night temperature accompanied by light rainfall or heavy dew and in fields previously sown with solanacous crops has also been reported (Kirk et al., 2013). Mohind (2001) has also reported prevalence of late blight disease in case of potato under Kashmir conditions. The data on infection of tomato fruits reveals that late blight of tomato is not strictly a foliar disease but affects fruits also. Out of infected fruits, a good proportion is unmarketable due to presence of large lesions or rot. Fruits infected severely were completely browned or get partially or fully rotten and are as such not accepted in the market. Such fruits are classified as unmarketable culls. Fruit infection and formation of unmarketable culls due to this disease has also been reported by many workers (Watterson, 1986; Tripathi et al., 
2017). Shah (2009) has also reported prevalence of Phytophthora fruit rot of tomato in varying degrees of incidence in all the surveyed locations of Kashmir.

Late blight (Phytophthora infestans) is more or less prevalent in almost every tomato field of Kashmir valley with varied incidence and severity from place to place. At green fruit stage, highest disease incidence of $13.71 \%$, intensity $(5.10 \%)$ and fruit infection $(5.08 \%)$ were recorded at Sagam, UttarasoNajigund and HilarArhama, respectively. Palhalan, Dawlatpora and Wager recorded the disease parameters (disease incidence, intensity and fruit infection) to moderate levels. Least disease incidence $(7.94 \%)$, intensity $(2.13 \%)$ and fruit infection $(2.14 \%)$ were recorded at Shangrin, Kaisarmulla and Palhalan, respectively. Similarly the data revealed that at ripe fruit stage highest disease incidence $(23.01 \%)$, intensity (10.19\%) and fruit infection $(9.35 \%)$ were recorded at Larkipora, HilarArhama and Haji Danter, respectively. Highest unmarketable culls (27.39\%) and least fruit yield $\left(140 \mathrm{qha}^{-1}\right)$ were recorded at Wagoora (Budgam). Kremshore, Wadura, Nowbugh and Wager recorded the disease parameters (disease incidence, intensity and fruit infection) to moderate levels. Least disease incidence $(17.47 \%)$, intensity $(7.13 \%)$ and fruit infection $(5.95 \%)$ were recorded at Bugam, Palhalan and Arampora, respectively. Least unmarketable culls $(21.16 \%)$ and highest fruit yield $\left(190 \mathrm{~kg}^{-1}\right)$ were recorded at Wadura (Baramulla).

\section{References}

Bose, K. S. and Agrawal B. K. 2007. Effect of lycopene from cooked tomatoes on serum antioxidents enzymes, lipid peroxidation rate and lipid profile in cronary heart disease. Singapore Medical Journal 48:415-420.

Drenth, A. Janssen, E. M. and Govers, F.
1995. Formation and survival of oospores of Phytophthora infestans under natural conditions. Plant pathology 44: 86-94.

Kirk, W., Wharton, P., Hammerschmidt, R., Abu-el Samen, F. and Douches, D. 2013. Late blight. Michigan State University Extension Bulletin No: E2945 pp. 03-04. Available on: http://www.potatodiseases.org/lateblight .html.

Mabbett, T. H. and Phelps, R. H. 1984. Effect of leaf growth in cucumber on deposit dilution and the control of angular leaf spot. Tropical Pest Management 30: 444-449.

Meya, A. I., Mamiro, D. P. and Kusolwa, P. 2015. Response of late blight disease resistant variety to common occurring tomato diseases in the field. Asian Journal of Plant Science Research 5: 815.

Mohid, A. R. 2001. Studies on potato late blight [Phytophthora infestans (Mont.) de Bary]. M. Sc. thesis pp. 77.

Niaka, S., De-Jude, J. 1., De-Goffau, M., Hilmi, M. and Van-Dam, B. 2005. Pest and disease. In: Cultivation of tomato (Ed. V. Barbara) pp. 37-55.

Rana, M. K. 2008. Olericulture in india. Kalayani publishers, New Delhi pp. 4399.

Sameera, S. 2007. On some aspects of plot technique in field experiments on tomato (Lycopersicon esculentum. Mill) in soils of Kashmir. M. Sc. Thesis pp. 12.

Shah, T. A. 2009. Cause and management of phytophthora fruit rot of tomato (Lycopersicon esculentum Mill.) in Kashmir. Ph. D Thesis pp. 1-224.

Sokhi, S. S., Thind, T. S. and Dhillon, H. S. 1993. Late Blight of potato and tomato. Punjab Agricultural University, Ludhiana. Directorate of research publication. pp. 19. 
Tripathi, A. N., Pandey, K. K., Meena, B. R., Rai, A. B. and Singh, B. 2017. An emerging threat of Phytophthora infestans causing late blight in tomato in Uttar Pradesh, India. New Disease Reports 35: 14.

Tsedaley, B. 2014. Late blight of potato (Phytophthora infestans) biology economic importance and its management approaches. Journal of Biology, Agriculture and Healthcare 4: 215-225.

Tulsane, L. R. 1854. Note on fungal endophytes such as that of the Potato.
Rendu Acadimic Sciences 38:1101-1104. Vale, F. X. R., Zambolium, L. and Paul, P. A. 2000. Controle de doencas de plantas pp. 173-207 In: Hortalicasvisconde do riobranco (Ed. H. Costa). Costa and paul Suprema Grafica e Editora p. 23.

Watterson, J. C. 1986. Diseases. In: The tomato crops. (Eds. Atherton and Rudich) Chapman and hall limited New York pp. 461-462.

Willcox, J. K., Catignani, G. L. and Lazarus, S. 2003. Tomatoes and cardiovascular health. Critical Reveiws in Food Science Nutrition 43:1-18.

\section{How to cite this article:}

Farheen Chasti, Nazir Ahmad Bhat, Roaf Ahmad Rather, Masrat Ul Nisar and Tanveer Ahmad Wani. 2018. Severity of Tomato Late Blight Caused by Phytophthora infestans (Mont.) De Bary in Kashmir. Int.J.Curr.Microbiol.App.Sci. 7(10): 3036-3047. doi: https://doi.org/10.20546/ijcmas.2018.710.354 